\title{
The effects of fall experience by tripping on the movement of stepping over an obstacle
}

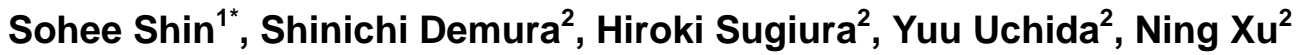 \\ ${ }^{1}$ Department of Sports Medicine and Sports Science, Graduate School of Medicine, Gifu University, Gifu, Japan; \\ *Corresponding Author: sohee@gifu-u.ac.jp \\ ${ }^{2}$ Graduate School of Natural Science \& Technology, Kanazawa University, Kanazawa, Japan
}

Received 21 January 2013; revised 21 February 2013; accepted 28 February 2013

Copyright (c) 2013 Sohee Shin et al. This is an open access article distributed under the Creative Commons Attribution License, which permits unrestricted use, distribution, and reproduction in any medium, provided the original work is properly cited.

\begin{abstract}
This study examines the effects of fall experience caused by tripping on the movement of stepping over an obstacle. The participants were divided into 3 groups ( 26 fallers caused by tripping, 24 fallers caused by other causes, and 145 non-fallers). Participants stepped forward over a $10 \mathrm{~cm}$ high obstacle with one leg, and then returned to their original position five times as quickly as possible. The OSFS (obstacle single leg forward step) test was measured in the following 2 phases: the OSFS-F phase, in which participants stepped out on one leg, and the OSFS-R phase, in which they returned it. Significant differences among the three groups were found in all parameters, and the fallers by tripping were significantly inferior to the non-fallers. There were no significant differences between the fallers by other reasons and the nonfallers in all parameters. The fallers by tripping are slower in the obstacle step movement than the non-fallers.
\end{abstract}

Keywords: Tripping; Faller/Non-Faller; OSFS Test

\section{INTRODUCTION}

Visual According to the reports of Suzuki et al. [1] and Demura et al. [2], which researched the actual conditions of falling in community-dwelling elderly people, a fall incidence rate for one year was about $20 \%$ in the elderly aged 65 and over, although there were some regional differences. In addition, because fall incidence increases with age, the possibility that the elderly will get fractures and become bedridden may also increase. A decline in physical fitness seriously limits daily life activities for the elderly, and it enhances the possibility of a fall. To help them avoid falling, it will be important to measure their physical fitness level adequately. Suzuki et al. [3] evaluated the life states and physical functions of community-dwelling elderly people using the Tokyo Metropolitan Institute of Gerontology Index, and reported that the fallers' fall risk scores were higher than the non-fallers'. Tinetti [4] also reported that the elderly with high activity level have a lower fall incidence. Hence, a decline in daily life activities may be closely related to fall risk.

There are mechanisms causing elderly falls, including trip, slip, misstep and stagger. Fall mechanisms and optimal prevention strategies differ respective to each. According to previous studies $[5,6]$ that reviewed causes, falling is mostly due to a trip during walking. Decreased leg strength and visual function makes foot lifting more difficult, and easily results in tripping; but this condition will also likely improve with training. On the other hand, falls caused by "sliding”, "staggering”, "dizziness", and "swaying" occur mostly because of environmental factors and medical history. Hence, an individual profile is necessary to effectively prevent falling [7]. Most falls occur when persons with internal factors, such as physical function and decreased performance of physical activities, are affected by external factors like an obstacle. Therefore, it is important to observe each person in detail, to understand what things become risk factors on an individual basis.

Until now, fall experience has been evaluated by whether or not a person has fallen previously, and it has been reported that the elderly with fall experience have a high risk of falling again [3]. In evaluating fall risk, past fall experience is important information, as are physical functional factors such as ADL (activities of daily living) and walking ability or environmental factors. On the other hand, many fall-related physical fitness tests have been developed to predict fall risk. To approach fall prevention from various view points, it is necessary to understand which body functions are evaluated by the re- 
sults obtained from field tests. For example, the elderly who cannot stand without swaying much also cannot maintain a stable posture, and thus may be predicted to fall down. In addition, the elderly with inferior leg strength are unable to support the body when stepping forward quickly and thus may be predicted to fall down.

Shin and Demura [8-12] focused on the movement of making a new base of support by stepping forward, developed the single leg forward step test (SFS test) which evaluates the elderly's fall-related physical fitness, and examined its utility.

Individuals avoid a fall by returning to a stable base of support (BOS), which is established by stepping forward quickly when sensing an impending fall. In the single leg forward step test, participants must step forward and return the stepped leg to the original position quickly. Hence, the supporting and stepping legs require considerable leg strength and balance ability, because braking power is needed to stop the body's forward momentum. It was reported that the test using this movement has a strong relationship to the fall risk score and is useful to evaluate the elderly's fall-related physical fitness [11]. Shin and Demura [12] reported that persons with fall experience in the past year were significantly inferior to persons without fall experience in the SFS test and the test related to ADL scores. It is assumed that when adding an obstacle in the above-mentioned SFS test, the elderly with fall experience by tripping will find it more difficult to complete the SFS test. If the elderly that trip easily are screened by the SFS test, in the future a more accurate approach may be able to offer fall prevention at an individual level.

This study examined the effects of fall experience caused by tripping on the movement of stepping over an obstacle.

\section{METHODS}

\subsection{Participants}

The participants were 195 healthy elderly women who can walk independently. The fallers with fall experiences in the past year numbered 50 subjects, and 26 subjects experienced falls by tripping. The rest of the subjects experienced falls for reasons other than tripping. "Slipping" and "Swaying" each numbered 11 subjects, respectively; "Fainting" and "Pushing by external force" were experienced by one subject. Participants were divided into the non-faller group (G1, 145 subjects), trip-faller group (G2, 26 subjects) and other-faller group (G3, 24 subjects). Prior to the various measurements, the purpose and procedure of this study were explained in detail and informed consent was obtained from all participants. This study was approved by the Ethics Committee on Human Experimentation of Faculty of Education, Kana- zawa University.

\subsection{Obstacle-Single Leg Forward Step Test (O-SFS Test, Figure 1)}

Participants stood barefooted with relaxed arms on a step sheet in a quiet room. They were asked to look at the obstacle. Before the measurement, it was determined which leg was easiest to stand on and operate, by Demura et al.'s assessment [7]. Participants stood with the supporting leg, stepped forward over the obstacle with the other leg, and returned to an original position five times as quickly as possible. The step width from the start spot was $25 \mathrm{~cm}$, and the obstacle (height: $10 \mathrm{~cm}$ ) was set at the midway point. The measurement was conducted in one trial after one practice trial, and the mean was used for statistical analysis. A tester controlled the obstacle lightly so that it did not move from the set place or fall down if participants tripped over it. The step test was performed using the step sheet (Takei Inc. Japan), which uses foot pressure to measure swing time in one leg from rising to landing. The short swing time means that participants were able to step quickly. Parameters were the time of a forwarding phase (OSFS-F: A mean time of stepping forward) and a returning phase (OSFS-R: A mean time of returning to the original place), and a mean time of both phases.

\subsection{Statistical Analysis}

To assess mean differences among the fallers by tripping, the fallers by other reasons, and the non-fallers in the OSFS test, the One-way analysis of variance was used. Multiple post hoc comparisons were performed using Tukey's HSD method if there were significant differences. The probability level of $p<0.05$ was indicative of statistical significance. Statistical program for the

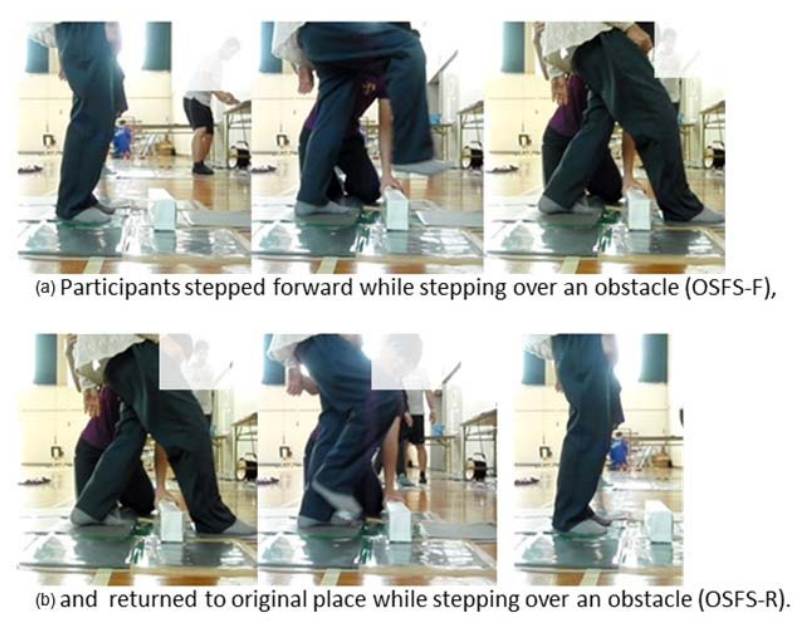

Note: Participants repeated the above measurement five times, as fast as possible.

Figure 1. Obstacle single leg forward step test (OSFS test). 
calculation was applied IBM SPSS statistics 19.

\section{RESULTS}

Table 1 shows the test results of mean differences for age, height and weight among the three groups: fallers by trip, fallers by other reasons and non-fallers. They showed no significant differences among groups.

Table 2 shows the test results of mean differences among groups for OSFS test parameters. There were significant differences in all parameters, and the fallers by tripping were significantly inferior to the non-fallers. There were no significant differences between the fallers by other reasons and the non-fallers in all parameters.

\section{DISCUSSION}

To evaluate the elderly with lower physical fitness, very safe tests should be selected. In addition, it is preferable that the test content relates closely to their daily life ac tivities and is available for rehabilitation and func- tional recovery [13]. This study set an obstacle in a general single leg forward step test and examined performance characteristics of the fallers by tripping.

Until now, fall risk has been evaluated based on fall experience in the previous year. If movements or mechanisms with high probability of fall incidence are presented in advance, the elderly may be able to easily understand "the movements during which they should be careful" or "the physical functions that they should improve to avoid falling”. For example, training methods to avoid tripping may be offered for the elderly who were screened as being likely to fall down by tripping. This study investigated fall experience in the past year, including the movements that led to the fall, and found that 26 of 50 fallers fell down by tripping. Although this number was a little fewer than that reported in previous study [14], it was clarified that many falls are attributed to tripping. Falls by tripping have a higher prevention possibility via improvement of daily physical activities or visual function than falls by reasons such as "slipping”,

Table 1. The test results of the mean differences between groups for age, height and weight.

\begin{tabular}{|c|c|c|c|c|c|}
\hline & & Mean & SD & F-value & P-value \\
\hline \multirow{2}{*}{ Years } & Other faller & 76.0 & 6.7 & 0.65 & 0.53 \\
\hline & Non-faller & 75.6 & 6.0 & - & - \\
\hline \multirow{3}{*}{$\begin{array}{l}\text { Height } \\
\text { (cm) }\end{array}$} & Other faller & 150.3 & 8.3 & 0.79 & 0.45 \\
\hline & Trip-faller & 148.9 & 6.0 & - & - \\
\hline & Non-faller & 148.4 & 7.5 & - & - \\
\hline \multirow[b]{2}{*}{$\begin{array}{c}\text { Weight } \\
\text { (kg) }\end{array}$} & Other faller & 51.9 & 8.4 & 0.59 & 0.55 \\
\hline & Trip-faller & 52.3 & 8.8 & - & - \\
\hline
\end{tabular}

Note-Trip: The faller by trip, Other: The fallers for reasons other than tripping.

Table 2. The test results of the mean differences between groups for the OSFS test.

\begin{tabular}{|c|c|c|c|c|c|c|c|}
\hline & & Mean & SD & F-value & P-value & $\begin{array}{r}\text { Mult } \\
\text { ( T }\end{array}$ & $\begin{array}{l}\text { rison } \\
\text { D) }\end{array}$ \\
\hline \multirow{3}{*}{$\begin{array}{l}\text { OSFS } \\
\text { (sec.) }\end{array}$} & Other faller & 0.61 & 0.13 & $5.99^{*}$ & 0.00 & \multicolumn{2}{|c|}{ Non-faller < Trip-faller } \\
\hline & Trip-faller & 0.66 & 0.18 & - & - & ES: & 0.73 \\
\hline & Non-faller & 0.57 & 0.11 & - & - & - & - \\
\hline \multirow{3}{*}{$\begin{array}{c}\text { OSFS-F } \\
\text { (sec.) }\end{array}$} & Other faller & 0.56 & 0.11 & $3.37^{*}$ & 0.04 & \multicolumn{2}{|c|}{ Non-faller $<$ Trip-faller } \\
\hline & Trip-faller & 0.62 & 0.19 & - & - & ES: & 0.54 \\
\hline & Non-faller & 0.56 & 0.11 & - & - & - & - \\
\hline \multirow{3}{*}{$\begin{array}{c}\text { OSFS-R } \\
\text { (sec.) }\end{array}$} & Other faller & 0.63 & 0.15 & $7.59^{*}$ & 0.00 & \multicolumn{2}{|c|}{ Non-faller $<$ Trip-faller } \\
\hline & Trip-faller & 0.69 & 0.20 & - & - & ES: & 0.81 \\
\hline & Non-faller & 0.58 & 0.12 & - & - & - & - \\
\hline
\end{tabular}

Note-Trip: The faller by trip, Other: The fallers for reasons other than tripping, ${ }^{*} \mathrm{p}<0.05$. 
"staggering”, “dizziness", "swaying” and etc. For five weeks, Weerdesteyn et al. [15] examined different approaches of the trip avoidance process, and reported that the success rate of stepping over an obstacle increased significantly by performing a unique program, including performing multiple tasks at once and other obstacle courses that simulate daily life. In addition, Lamoureux et al.'s [16] twenty-four week study showed that the elderly's obstacle avoidance ability improved significantly by resistance exercises, including flexion and extension movements of the hip, knee and ankle joints.

This study divided the causes of falls into "tripping" and "reasons other than tripping", and examined the characteristics of the fallers using the step movement. The step movement was simple because participants stepped forward over a $10 \mathrm{~cm}$ high obstacle on one leg and returned it to the original position. It was found that the fallers by tripping take longer than the non-fallers and the fallers for reasons other than tripping. The OSFS test was made up by considering the following four points: 1) when almost falling, the elderly step forward one step to keep their base of support (BOS) and to prevent the fall; 2) a decrease in leg strength and balance ability as well as the range of motion (ROM) of the hip, knee and ankle joints is connected with factors related to the fall; 3) the elderly fall frequently by tripping over an obstacle or a step; and 4) the screening test should use movements which the elderly can easily understand. The OSFS test asks the elderly to step over the obstacle while supporting the body on one leg and to return it to original position again. Hence, it may be difficult for the elderly with decreased physical fitness, as compared with walking and one-leg standing.

When the OSFS was divided by both phases, the fallers by tripping were inferior to the non-fallers in the OSFS-F (the phase of stepping forward) and OSFS-R (the phase of returning to the original place). Therefore, a fall risk may be better explained by the movement (OSFS-R) in returning the stepped leg to the original place than the movement in stepping over an obstacle (OSFS-F).The elderly with decreased leg strength due to a sense of fear of falling forward cannot stretch legs when a body inclined forward [17]. A lack of the ability to brace one's legs may cause falls.

Meanwhile, the OSFS-F phase also showed a significant difference. The fallers by tripping may take a long time to step over an obstacle regardless of the phase, OSFS-F or OSFS-R. Watanabe [18] reported that the elderly are more likely to disrupt body balance in the conditions which require posture change, such as stepping over obstacles. Namely, the elderly that trip easily used a higher clearance than the obstacle because they cannot elevate the leg smoothly by the dorsiflexion and flexural movement of the hip, knee, ankle joints. In addi- tion, such elderly people may find it difficult to maintain physical balance on one standing leg while stepping over the obstacle. Hence, the elderly may have needed a longer time when shifting to the next movement after stepping forward or after returning to the original place.

\section{REFERENCES}

[1] Suzuki, T. (2000) Questionnaire for falls assessment of elderly people and its application. Health Assessment Manual Kosei Kagaku Kenkyusho, Tokyo.

[2] Demura, S., Sato, S. and Sugiura, H. (2010) Lower limb laterality characteristics based on the relationship between activities and individual laterality. Gazzeta Medica Italiana, 169, 181-191.

[3] Suzuki, T. (2003) Epidemiology and implications of falling among the elderly, Nippon Ronen Igakkai Zasshi, 40, 85-94. doi:10.3143/geriatrics.40.85

[4] Tinetti, M.E. (1986) Performance oriented assessment of mobility problems in elderly patients. Journal of American Geriatrics Society, 34, 119-126.

[5] Suzuki, T. (2001) Fall accidents of the elderly. Journal of Clinical Rehabilitation, 10, 955-960.

[6] Yasumura, S. and Shinno, N. (1997) Risk factors for falls among elderly people. Journal of physical therapy, 14, 199-205.

[7] Demura, S., Sato, S., Shin, S. and Uchiyama, M. (2012) Setting the criterion for fall risk screening for healthy community-dwelling elderly. Archives of Gerontology and Geriatrics, 54, 370-373.

doi:10.1016/j.archger.2011.04.010

[8] Shin, S. and Demura, S. (2007) Effective tempo of the step test for dynamic balance ability in the elderly. Journal of Physiological Anthropology, 26, 563-567. doi:10.2114/jpa2.26.563

[9] Shin, S. and Demura, S. (2009) The relationship of age and leg strength in the step test with stipulated tempo in the elderly. Archives of Gerontology and Geriatrics, 49, 311-316. doi:10.1016/j.archger.2008.11.009

[10] Shin, S. and Demura, S. (2009) Relationship between the step test with stipulated tempos and gait ability in the elderly. Journal of Physiological Anthropology, 28, 4954. doi:10.2114/jpa2.28.49

[11] Shin, S. and Demura, S. (2010) The usefulness of single leg forward step test for the evaluation of the elderly's ADL ability. Journal of Physiological Anthropology, 10, 27-32.

[12] Shin, S. and Demura, S. (2010) Comparison and agelevel differences among various step tests for evaluating balance ability in the elderly. Archives of Gerontology and Geriatrics, 50, 51-54. doi:10.1016/j.archger.2009.05.007

[13] Demura, S., Shin, S. and Yamaji, S. (2008) Sex and age differences of relationships among stepping parameters for evaluating dynamic balance in the elderly. Journal of Physiological Anthropology, 27, 207-215. doi:10.2114/jpa2.27.207 
[14] Akiyama, T., Fukushima, T., Hisasita, H. and Ebina, H. (1996) Study on own-injuries of the elderly in outdoor walk space. The report of Japan Society of Traffic Engineers, 16, 193-196.

[15] Weerdesteyn, V., Rijken, H., Geurts, A.C., Smits-Engelsman, B.C., Mulder, T. and Duysens, J. (2006) A fiveweek exercise program can reduce falls and improve obstacle avoidance in the elderly. Gerontology, 52, 131-141. doi:10.1159/000091822
[16] Lamoureux, E., Sparrow, W.A., Murphy, A. and Newton, R.U. (2003) The effects of improved strength on obstacle negotiation in community-living older adults. Gait and Posture, 17, 273-283.

doi:10.1016/S0966-6362(02)00101-7

[17] Mano, Y. (2008) Falls in the elderly and measurements. Ishiyaku Press, Tokyo.

[18] Watanabe, K. (1993) A response to an obstacle in when the elderly walk. Health care, 35, 93-97. 